\title{
Characters Style application Based Convolutional Neural Network
}

\author{
Haiyan Deng ${ }^{1, a)}$, Yijun Liu $^{2}$, b) \\ ${ }^{1}$ School of Computers. Guangdong University of Technology. Guangdong, Guangzhou, China \\ ${ }^{2}$ School of Information Engineering. Guangdong University of Technology. Guangdong, Guangzhou, China \\ a) peptreltongxu@163.com \\ b)yjliu@gdut.edu.cn)
}

\begin{abstract}
In the past ten years, convolutional neural networks have achieved a series of breakthrough developments in the areas of classification detection, pattern recognition, and semantic segmentation. Convolutional neural network learning has led to a wide range of applications and research for its capabilities and feature extraction and classification recognition capabilities. And it has important analysis and research value. Firstly, the historical background and the development status of convolutional neural network is introduced. The following are basic structure and operation principle of convolutional neural network. What's more, the network structure of Chinese character application based on convolutional neural network are mainly introduced. Finally, we summarize the current deficiencies of the convolutional neural network and the future direction of development.
\end{abstract}

Key words: characters; deep convolutional neural networks; image generation; network structure.

\section{INTRODUCTION}

In recent years, the structure of convolutional neural networks study is still in a stage of rapid development, and some network structures with excellent performance have been proposed[1,2].The single-layer perceptron model was come up with in the 20th century, until the wide application of the neural networks and the zero-failure record of AlphaGo Zero, a series of achievements made by artificial intelligence fully demonstrate the great potential of deep learning neural networks, with respect to the Artificial neural networkable simple convolutional neural network structure, LeNet and these currently popular deep learning models such as deep belief network(DBN),Stacked Denoising Autoencodes (SDA),convolutional neural network(CNN).At the beginning of 1980 s, research scholars proposed CNN research work and achieved good results in handwritten digit recognition, and achieved successful application of the transfer learning theory on convolutional neural networks. The application of the neural network has been further extended [3]. Then gradually applied to detection and recognition and the generation of Chinese characters [3-12]. The research results of convolutional neural networks emerging in various fields have made it one of the most popular research hotspots.

\section{RELATED WORK}

\section{Convolution neural networks}

Convolution neural networks provide an end-to-end learning model, which can be trained by the traditional gradient descent method. The trained convolution neural network can extract the features from the training datasets, and it can achieve the extraction and classification according to training features. Perceptron was come up by Frank Rosenblatt in 1957, which was the convolution network and the ancestor of the neural network. In the 20th century 80 years, Japanese scientist Kunihiko Fukushima proposed a multi-level neural network named Neocognitron, which had a certain visual cognitive function and directly inspired the subsequent convolution neural network. LeNet-5 was proposed by LeCun in 1997, the first proposed multi-level cascade 
convolution structure, which can identify handwritten numbers effectively. In 2012, Alex adopted 8 layers convolution neural network AlexNet [4] --The neural network, which has 60 million parameters and 650,000 neurons, consists of five convolutional layers, some of which are followed by max-pooling layers, and three fully-connected layers with a final 1000-way softmax. AlexNet won the championship in ILSVRC 2012 and set off the boom of the convolutional neural network.

Since convolutional neural network has developed, the structure varies from the field of handwriting recognition in the field of LeNet-5 to rhe network, which won the championship Krizhevsky in the accuracy beyond the second place $11 \%$ of the great advantage about the large-scale image database ImageNet 19 image classification contest, and others proposed AlexNet, and then the later new convolution of the neural network model, such as Oxford University VGG (Visual Geometry Group),Google's GoogLeNet, Microsoft's ResNet, Goodfellow, Ian, et al advanced GAN[5] "Generative adversarial nets, etc. And convolution Neural networks are continually integrated with some traditional algorithms, such as migration learning methods, making the application of convolution neural networks to more fields. From the initial simpler handwriting character recognition, and gradually extended to some of the more complex areas, such as pedestrian detection ", behavior recognition", "human body posture recognition, natural language processing",, Speech recognition one, etc. What's more, GAN can be applied to create interactive programs to help users create much more realistic images with corresponding-outline what they imaged. And GAN don't need any Monte Carlo approximations to train, it also fit the data distribution, the generator parameters are updated, not from the data samples, but the back-propagation of the discriminator.

Convolutional neural network is a special type of deep feedforward neural network, mainly composed of input layer, hidden layer, fully connected layer and output layer. The hidden layer is alternately connected by convolution layer and down sampling layer. A classic convolutional neural network model is shown in Figure 1. The model consists of seven layers, including an input layer, two convolutional layers, two sampling layers, a fully connected layer, and an output layer. The convolutional layer extracts the features through the convolution operation and then extracts them through the down sampling operation.

The features are combined into more abstract features, then the combined features are input into one or more fully connected layers, and each neuron of the full connected layer is fully connected with all neurons of the previous layer. The last full connection layer connects to the output layer.

\section{CNN analysis of the Chinese characters}

The classic CNN network structure is commonly used in handwritten data sets--MNIST. Nowadays many researchers approach to combine CNN models with recognition of the Chinese characters, all of them adopt convolutional network to learn the characters. Some papers show that DCNN have better performance in Chinese characters [6-8]. For example, in blog [9] exploring the Latent Space of Chinese Handwriting by a deep convolutional generative adversarial network (DCGAN)with training the Handwriting database. Baoyao Zhou, et [10] put forward an efficient solution to generate Chinese handwritten fonts by effectively reusing the sample characters that users write.Jeng-Wei Lin, et[11]presented an easy and fast solution for an ordinary user to create a Chinese font of his or her handwriting style. Peng Liu, et [12] proposed automatic Generation of Personalized Chinese Handwriting Characters through an example-based approach. By imitating shapes of individual character components as well as the spatial relationships between them, the proposed method can automatically generate personalized handwritings following an example-based approach.

\section{THE NETWORK STRUCTURE}

In ICDAR handwritten Chinese character recognition competition,the data sets include HCL2000 [13] of Beijing University of Posts and Telecommunications, HIT. MWl [14] of Harbin Institute of Technology and so on. And most of them use CNN to classify and recognize after learning features. Some applications are adopting convolutional neural network (CNN) [7,15,16,17,18], Recurrent Neural Network(RNN) [19,20], generation adversarial network(GAN) [5,21,22].and so on.

For example, the earlier application in paper [18], CNNs are applied in handwritten character recognition, attempts to use CNN-connected component detectors and CNN shadow detectors to get the characteristics of handwritten Japanese characters. For [23], the size of 10 input images are 48x48, the structure of CNN:100C3-MP2-200C2-MP2-300C2-MP2-400C2-MP2$1000 \mathrm{~N}-3755 \mathrm{~N}$.where $100 \mathrm{C} 3$ means 100 filters of size $3 \times 3$ Convolutional layer, and MP means MAX Pooling, Finally, the fully connected output layer has 3755 neurons and each neuron corresponds to one-character class. Paper [24] present an effective method to analyze the recognition confidence of handwritten Chinese characters. The architecture of the network is represented as follows: 96×96Input-100C3-MP2-200C2-MP2-300C2-MP2-400C2-MP2-500C2-MP2-600C2-1024FC-10081Output. What's more, the paper adopts ReLU non-linearity as activation function between convolutional layer and pooling layer [4]. Meanwhile, dropout and batch normalization, as important and effective methods proposed by [25] [26].

Another popular methods are GAN. For instance, [25] advanced CGAN generated MNIST samples, In the generated network model, there are two models, a network generation model G, and a discriminant model D. Network G is used to generate some random noise by input some images and Network $\mathrm{D}$ is used to determine if the input is a generated image or we Your own image in the database. Both $\mathrm{G}$ and $\mathrm{D}$ can be some multi-layered network input, we usually use CNN or RNN to handle this. 


\section{DEVELOPMENT DEFECTS AND DIRECTIONS}

The study of the structure of convolutional neural networks also has a lot of room. Current research shows we can increase the complexity of the character generation network to solve some problems such as overfitting and network degradation. Convolutional neural network performance needs to rely on a more reasonable and efficient network structure design.

The parameters of convolutional neural network are numerous, but the current related settings are mostly derived from practical experience. Optimization analysis of parameters and evaluation of network structure are a problem to be solved in convolutional neural networks.

The model structure of convolutional neural networks is continuously improving, and the number and diversity of data sets are more demanding. The complexity of data sets and the complexity of the network are closely related to the hardware and software conditions at that time.

Other learning theories, like Migration learning and adversarial learning, may can help to further expand the development of convolutional neural network applications, and combined convolutional neural networks with other methods, it's one of the current trends to help improve the real-time nature of the network.

\section{CONCLUSION}

In this paper, we sum up and analysis CNN for Chinese character research areas. And the papers showed the architecture is suitable for corresponding application, no matter for generation or recognition. Further, there are constantly new technologies proposed, like a new Convolutional Neural Network can help us generate our Chinese font database, which had achieved outstanding generation effect for Chinese characters.

\section{ACKNOWLEDGMENTS}

The paper is supported by funds from Department of Science and Technology at Guangdong Province and Guangzhou City (No.201604010051,2015B090901060, 2016B090903001,2016B090904001,2016B090918126, 2016KZ010101).

\section{REFERENCES}

1. K. Simonyan and A. Zisserman. Very deep convolutional networks for large-scale image recognition. arXiv preprint arXiv: 1409.1556,2014.

2. HE K, ZHANG X, REN S, et al. Deep residual learning for image recognition [EB / OL]. [2016-01-04]. https: / / www.researchgate.net / publication /286512696_ Deep_Residual_Learning _ for_Image_Recognition.

3. COLLOBERT R, WESTON J, BOTTOU L, et al. Natural language processing (almost) from scratch Journal of Machine Learning Research,2011,12(1): 2493-2537.

4. Krizhevsky, Alex, Sutskever, Ilya, and Hinton, Geoff. Imagenet classification with deep convolutional neural networks.In Advances in Neural Information Processing Systems 25,pp.1106-1114,2012.

5. Ian Goodfellow, Jean Pouget-Abadie, Mehdi Mirza,Bing Xu, David Warde-Farley, Sherjil Ozair, Aaron Courville, and Yoshua Bengio. Generative adversarial nets. In NIPS, pp.2672-2680.2014.

6. C.-L. Liu, S. Jaeger, and M. Nakagawa, "Online recognition of Chinese characters: The state-of-the-art," IEEE Trans. Pattern Analysis and Machine Intelligence, vol.26, no.2, pp.198-213,2004.

7. Z. Zhong, L. Jin, and Z. Xie, "High performance offline handwritten Chinese character recognition using GoogLeNet and directional feature maps," Proc. Int'l Conf. Document Analysis and Recognition (ICDAR), 2015.

8. W. Yang, L. Jin, D. Tao, Z, Xie, and Z. Feng, "DropSample: A new training method to enhance deep convolutional neural networks for large-scale unconstrained handwritten Chinese character recognition," arXiv: 1505.05354, 2015.

9. http://genekogan.com/works/a-book-from-the-sky/.2015 December 15.

10. Baoyao Zhou, Weihong Wang, Zhanghui Chen, EASY GENERATION OF PERSONAL Chinese HANDWRITTEN FONTS. 978-1-61284-350-6/11/\$26.00.2011 IEEE.

11. Jeng-Wei Lin, Chian-Ya Hong, Ray-I Chang, Yu-Chun Wang, Shu-Yu Lin. Complete Font Generation of Chinese Characters in Personal Handwriting Style.978-1-4673-8590-9/15/\$31.00 C2015 IEEE.

12. Peng Liu, Songhua $\mathrm{Xu}$, Shujin Lin, Automatic Generation of Personalized Chinese Handwriting Characters.978-0-7695-4899-9/12 \$26.00 C 2012 IEEE DOI 10.1109/ICDH.2012.77.109-116. 
13. Yu JinHui, Peng QunSheng, "Realistic synthesis of cao shu of Chinese calligraphy," Computer Graphics, 2005, vol.29, pp.145-153.

14. Xu Songhua, Lau, C. M. Francis, Cheung, K. William and Pan, Yunhe. "Automatic generation of artistic Chinese calligraphy," IEEE intelligent systems, 2005, pp.32-39.

15. D. Ciresan and J. Schmidhuber, "Multi-column deep neural networks for offline handwritten Chinese character classification," arXiv: 1309.0261,2013.

16. C. Wu, W. Fan, Y. He, J. Sun, and S. Naoi, "Handwritten character recognition by alternately trained relaxation convolutional neural network," Proc. Int'l Conf. Frontiers in Handwriting Recognition (ICFHR), pp. 291-296,2014.

17. L.Chen, S. Wang, W. Fan, J. Sun, and S. Naoi, "Beyond human recognition: A CNN-based framework for handwritten character recognition," Proc. Asian Conf. Pattern Recognition (ACPR),2015.

18. H Suzuki ,T Matsumoto ,LO Chua.A CNN handwritten character recognizer. International Journal of Circuit Theory \& Applications.2010,20(5):601-612.

19. XY Zhang, F Yin, YM Zhang, CL Liu, Y Bengio. Drawing and Recognizing Chinese Characters with Recurrent Neural Network. IEEE Transactions on Pattern Analysis \& Machine Intelligence.2016, PP (99):1-1.

20. “enerating online fake Chinese characters with LSTM-RNN,"015. [Online]. Available: http:// blog. otoro. net/2015/12/28/recurrentnet-dreams-up-fake-chinese-characters-in-vector-format-with-tensorflow.

21. E. Denton, S. Chintala, A. Szlam, and R. Fergus, "Deep generative image models using a Laplacian pyramid of adversarial networks," arXiv:1506.05751,2015.

22. A. Radford, L. Metz, and S. Chintala, "Unsupervised representation learning with deep convolutional generative adversarial networks," arXiv:1511.06434,2015.

23. Y WangX Li,C Liu, X Ding,Y Chen.An MQDF-CNN Hybrid Model for Offline Handwritten Chinese Character Recognition.14th International Conference on Frontiers in Handwriting Recognition,2014.

24. Meijun He, Shuye Zhang, Huiyun Mao, Lianwen Jin. Recognition Confidence Analysis of Handwritten Chinese Character with CNN.

25. G.E. Hinton, N. Srivastava, et al, improving neural networks by preventing co-adaption of feature detectors, ArXiv preprint arXiv: 1207.0580,2012.

26. S.Ioffe, C. Szegedy, Batch normalization: accelerating deep network training by reducing internal covariate shift, arXiv: 1502.03167,2015.

27. Jia Jia. Research on Font Generation Method Based on Conditional Confrontation Network. Communication Design and Application.2017, 12. 